\title{
Insect Sting Reactions and Specific IgE to Venom and Major Allergens in a General Population
}

\author{
Holger Mosbech ${ }^{a} \quad$ Line Tang $^{b} \quad$ Allan Linneberg ${ }^{b-d}$ \\ ${ }^{a}$ Allergy Clinic, Copenhagen University Hospital Gentofte, Hellerup, ${ }^{\text {b }}$ Research Centre for Prevention and Health, \\ and ${ }^{\mathrm{C}}$ Department of Clinical Experimental Research, Rigshospitalet, Glostrup, and ${ }^{\mathrm{d}}$ Department of Clinical Medicine, \\ Faculty of Health and Medical Sciences, University of Copenhagen, Copenhagen, Denmark
}

\section{Key Words}

Cross reactions - Hymenoptera venom - Insect sting allergy . Specific IgE - Tryptase

\begin{abstract}
Background: Insect sting reactions are frequently reported, but population studies documenting the frequency and the relation to lgE-sensitization and serum tryptase are scarce. Methods: Questionnaire data and results from measurements of specific lgE against venom, major allergens and cross-reacting carbohydrate determinants (CCDs) were collected from 2,090 adult participants in a cross-sectional survey. Results: $13 \%$ of the population reported symptoms of sting reactions and about half were systemic in nature. In all, $15 \%$ were sensitized to venom but only $31 \%$ of these had reacted to stings and only $38 \%$ of those with reactions had IgE to venom. In addition, $12 \%$ with IgE to venom were double-sensitized (DS), i.e. to both bee and wasp venom. Among DS IgE to major venom allergens, rApi $m$ 1, rVes $v 1$ and $r$ Ves v 5 were negative and of no help in $31 \%$, but $59 \%$ could be identified as likely sensitized to bee or wasp. IgE to CCDs occurred in only $0.7 \%$, but $80 \%$ of these were DS. Finally, $36 \%$ with IgE to CCDs had had symptoms, mostly local. Serum
\end{abstract}

tryptase was not associated with a history of sting reactions. Conclusions: In a temperate climate, self-reported insect sting reactions and sensitization to venom are frequent, but in most cases, these are not seen in the same individual. In DS individuals, measurements of IgE to major allergens can be helpful in some but not all cases and additional analyses are needed. IgE to CCDs may have some clinical relevance.

(c) 2016 S. Karger AG, Basel

\section{Introduction}

Most people are exposed to insect stings several times during their lifetime. In the European temperate climate, wasps and honeybees from the Hymenoptera order are often the offenders. While the majority of stings just result in pain and limited swelling, some individuals get large local swellings, and in a small number, more severe systemic reactions occur. The prevalence of such reactions in the general population is only scarcely described $[1,2]$.

A demonstration of sensitization to insect venom is needed to make a proper allergy diagnosis, i.e. for the documentation of an allergic mechanism and to safely iden-

\section{KARGER}

E-Mail karger@karger.com

www.karger.com/iaa
(C) 2016 S. Karger AG, Basel

$1018-2438 / 16 / 1703-0194 \$ 39.50 / 0$
Correspondence to: Dr. Holger Mosbech

Allergy Clinic, Copenhagen University Hospital Gentofte

Kildegaardsvej 28

DK-2900 Hellerup (Denmark)

E-Mail hmos0006@geh.regionh.dk 
tify the offending insect. Such identification is crucial when allergen-specific immunotherapy is considered. Allergenic cross-reactivity with or without clinical relevance is a major challenge in this context [3]. Elevated serum tryptase has been associated with more severe insect sting reactions $[4,5]$, but it is unknown if it is a risk factor for such reactions in the general population.

The aim of this study was to estimate the prevalence of insect sting reactions in a general population based on questionnaires. In addition, the study aims to compare the results with levels of specific IgE to venoms, to venom major allergens and to cross-reacting carbohydrate determinants (CCDs) in the same individuals, focusing on clinical and immunological cross-reactivity and the influence of tryptase level.

\section{Material and Methods}

\section{Study Design}

The study was part of a cross-sectional population survey, including serum-specific IgE and tryptase determinations and structured questionnaires. It was based on the five-year follow-up of the Health2006 cohort. A detailed description of the baseline examination has been published elsewhere [6]. The participants in the baseline Health2006 cohort were drawn as a random sample from the background population aged 18-69 years, living in 11 municipalities in suburban Copenhagen. A total of 3,471 individuals (44.7\% of the sample) entered the study and participated in the health examinations, which took place between June 2006 and June 2008. In 2011-2012, participants in the baseline Health2006 were invited for a 5-year follow-up examination, including essentially the same study protocol. A total of 3,405 were eligible for invitation (21 had emigrated and 45 had died). A total of 2,308 (45.8\% men) agreed to participate and were re-examined between November 2011 and November 2012 [7]. The median age was 55.7 (range 24-76) years. For 88 participants, the serum sample left for analysis was not sufficient for all of the planned in vitro measurements and for a further 30 , a valid questionnaire was not available. Thus, data from both questionnaires and the in vitro measurements were available for 2,090 individuals. In this group, the median age of 55.7 years (range $24-76$ years) and gender ( $46.0 \%$ men) did not differ significantly from the total participating group.

\section{Structured Questionnaires}

The structured questionnaire was self-administered and included questions on various health, lifestyle and socioeconomic aspects. The insect-related questions were 'Did you ever have a swelling of more than $10 \mathrm{~cm}$ from side to side after a sting from a wasp or bee? (Yes/No)', 'Did you ever have an itching rash on larger areas of the body after a sting from a wasp or bee? (Yes/No)', 'Did you ever feel malaise with the need to lie down or with breathing problems after a sting from a wasp or bee? (Yes/No)' and 'If you had any of these symptoms, which insect(s) was/were responsible: wasp? (Yes/No), bee? (yes/no), unknown insect? (Yes/No)'. Having had an insect sting reaction was defined as being able to confirm at least one of the symptoms mentioned above.

Insect Stings in the General Population

\section{Serum Specific IgE}

Sera were stored frozen until tested. All sera were tested for IgE to honeybee venom (i1), venom from wasp species (i3), the recombinant major allergens rVes v 1 (i211), rVes v 5 (i209) and rApi $\mathrm{m} 1$ (i208) if IgE to venom was $\geq 0.10 \mathrm{kU}_{\mathrm{A}} / \mathrm{l}$, the isolated glycan part from bromelain (o214), as a CCD marker, and a screening mixture of inhalant allergens (Phadiatop ${ }^{\circledR}$ ) using the commercially available UniCAP 250 system (Phadia Diagnostics, Uppsala, Sweden, now Thermo Fisher Scientific). Serum tryptase was measured with an ImmunoCAP ${ }^{\circledR}$ tryptase assay, which determines total tryptase, i.e. pro-forms and mature forms of $\alpha$ - and $\beta$-tryptase. In a study by the manufacturer, the 95th percentile was $11.4 \mu \mathrm{g} / \mathrm{l}$ in 126 healthy individuals (aged 12-61 years; $48 \%$ males). This value has been adopted as the upper reference level in several insect allergy studies [4, 8].

\section{Ethical Issues}

All individuals gave written consent to participate in the studies, which were approved by the corresponding Institutional Review Boards (Ethics Committee of the Capital Region of Denmark, code H-3-2011-081), and conformed to the current Helsinki Declaration.

\section{Statistical Analyses}

The $\chi^{2}$ and the Fisher's exact tests were, where appropriate, used to investigate the association between categorical variables. Logistic regression without adjustment was applied to assess the odds ratio (OR) for the outcome between exposure groups.

\section{Results}

\section{Questionnaire Data}

In all, $13 \%(\mathrm{n}=272)$ of the population had experienced reactions to stings (table 1 ). The majority reported large local reactions (51\% were large local reactions only), while about one third reported rash and one third reported malaise. The pattern of reactions was similar for bee and wasp stings.

More individuals reported reactions to wasp stings ( $\mathrm{n}=203 ; 9 \%)$ than to stings from bees $(\mathrm{n}=83 ; 4 \%)$ and unknown insects $(\mathrm{n}=38 ; 2 \%)$. Finally, $2.5 \%(\mathrm{n}=54)$ reported reactions to both bees and wasps.

\section{in vitro Data}

In parallel to the reported clinical reaction pattern, more individuals had $\operatorname{IgE} \geq 0.35 \mathrm{kU}_{\mathrm{A}} / 1$ to wasp than to bee venom (13.6 and 3.3\%, respectively), with a range $<0.35-$ 67.5 and $<0.35-15.8 \mathrm{kU}_{\mathrm{A}} / \mathrm{l}$, respectively. $\mathrm{IgE}$ to both bee and wasp venom was seen in $1.8 \%$ and $84.9 \%$ had no $\operatorname{IgE}$ to the venoms.

A total of $30 \%$ of the population had specific IgE $\geq 0.35$ $\mathrm{kU}_{\mathrm{A}} / \mathrm{l}$ to airborne allergens (Phadiatop). This was significantly associated with being sensitized (i.e. having specific IgE) to insect venom: $67.1 \%$ of the bee-sensitized 
Table 1. Questionnaire data based on answers from 272 of the 2,173 individuals (13\%) who reported abnormal reactions to insect stings

\begin{tabular}{llllll}
\hline Stinging insect $^{1}$ & \multicolumn{3}{l}{ Reaction, $\mathrm{n}(\%)$} & & \\
\cline { 2 - 6 } & $\begin{array}{l}\text { large local } \\
\text { reaction (only) }\end{array}$ & $\begin{array}{l}\text { generalized rash } \\
\text { (without malaise) }\end{array}$ & malaise & $\begin{array}{l}\text { systemic reaction } \\
\text { (rash or/and malaise) }\end{array}$ & $\begin{array}{l}\text { at least } 1 \\
\text { reaction }\end{array}$ \\
\hline Wasp & $104(51)$ & $58(29)$ & $68(34)$ & $99(49)$ & $203(100)$ \\
Bee & $43(52)$ & $23(28)$ & $28(34)$ & $40(48)$ & $83(100)$ \\
Unknown & $19(50)$ & $11(29)$ & $11(29)$ & $19(50)$ & $38(100)$ \\
\hline
\end{tabular}

${ }^{1}$ More than one insect could be recorded for each individual.

[OR 5.4; 95\% confidence interval (CI) 3.3-8.9] and 47.2\% of the wasp sensitized were sensitized to airborne allergens as well (OR 2.6; 95\% CI 2.0-3.3; p < 0.0001).

Only $14 \%(10 / 73)$ of the individuals sensitized to bee venom had IgE to the major allergen (rApi m 1) $\geq 0.35$ $\mathrm{kU}_{\mathrm{A}} / 1$. In wasp venom, $35 \%$ of those sensitized to the venom $(103 / 303)$ had IgE to rVes v 1, 63\% (186/303) to rVes v 5 and $82 \%(248 / 303)$ to at least one of these. IgE to CCDs could partly explain the discrepancies since this IgE occurred in $19 \%(12 / 63)$, with $\operatorname{IgE}$ to bee venom but not to the major allergens, and in $11 \%(6 / 55)$ to wasp venom but not to the major allergens, compared with $10 \%(1 / 10)$ and $2.4 \%(6 / 248)$ in individuals with IgE to venom as well as to major allergens.

In addition, $12 \%(40 / 336)$ of the individuals with positive sera were positive to both venoms, i.e. they were double-sensitized (DS). Having IgE to one venom increased the risk of having IgE to the other (OR 8.7; 95\% CI 5.414.0). About half of the DS had IgE to major allergens from one of the species only, indicating either bee or wasp as the primary sensitizer (table 2). In a third of the DS, no IgE to the major allergens was found and the diagnosis was left open. In contrast to IgE against bee and wasp venom in the population, IgE against the major bee and wasp allergens in the DS did not correlate.

The occurrence of specific IgE to common CCDs in the venom extracts might, at least partly, explain the double sensitization, since $80 \%$ with IgE to CCDs were DS compared to only $1.3 \%$ with no IgE (table 3 ). Additionally, $50 \%(6 / 12)$ of the DS with no IgE to major allergens had IgE to CCDs compared to $22 \%(6 / 27)$ of the DS with IgE to at least one major allergen.

\section{Questionnaire and in vitro Data}

There was a correlation between sensitization and clinical symptoms; however, only $38 \%$ of the individuals
Table 2. Specific IgE to venom major allergens from bee (rApi m 1) and wasp (rVes v 1 and rVes v 5) in individuals with IgE to both bee and wasp venom, i.e. showing double sensitization

\begin{tabular}{llll} 
& \multicolumn{2}{c}{ rApi m 1} & \multirow{2}{*}{ Total } \\
\cline { 2 - 3 } & \multicolumn{1}{c}{ positive $^{1}$} & negative & \\
\hline rVes v 1 and/or rVes v 5 & & \\
$\quad$ Positive & $4(10 \%)$ & $21(54 \%)$ & $25(64 \%)$ \\
Negative & $2(5 \%)$ & $12(31 \%)$ & $14(36 \%)$ \\
\hline Total & $6(15 \%)$ & $33(85 \%)$ & $39(100 \%)$
\end{tabular}

${ }^{1}$ Specific $\operatorname{IgE} \geq 0.35 \mathrm{kU} / \mathrm{A}$. $\mathrm{p}=1.000$, Fisher's exact test.

reporting abnormal sting symptoms (to any insect) had $\operatorname{IgE} \geq 0.35 \mathrm{kU}_{\mathrm{A}} / \mathrm{l}$ to at least one venom (table 4 ). Excluding individuals with IgE to CCDs would change the frequency to $37 \%$.

Only $31 \%$ of the individuals with $\operatorname{IgE} \geq 0.35 \mathrm{kU}_{\mathrm{A}} / \mathrm{l}$ to at least one venom reported sting reactions/symptoms (to any insect; table 4). Excluding individuals with IgE to CCDs would change the frequency to $30 \%$.

In addition, 11 of the 14 individuals with specific $\operatorname{IgE}$ to CCDs $\geq 0.35 \mathrm{kU}_{\mathrm{A}} / \mathrm{l}$ and clinical data available were DS. Four of these $(36 \%)$ reported sting reactions. This reaction rate did not differ from that of individuals sensitized to at least one insect venom. All 4 had large local swellings and 1 had malaise and generalized rash in addition to the swelling. Two people with IgE to CCDs, but not to venom, had no symptoms related to the stings.

Among individuals with low IgE to venom (0.20-0.35 $\left.\mathrm{kU}_{\mathrm{A}} / \mathrm{l}\right)$ and reporting either generalized rash and/or malaise, 3 of $71(4 \%)$ had $\operatorname{IgE} \geq 0.35 \mathrm{kU}_{\mathrm{A}} / \mathrm{l}$ to the major wasp allergens. A similar ability to identify individuals sensi- 
Table 3. Specific IgE to CCDs in individuals with and without specific IgE to both bee and wasp venom $\geq 0.35 \mathrm{k} \mathrm{U}_{\mathrm{A}} / 1$

\begin{tabular}{|c|c|c|c|c|c|c|c|c|}
\hline & \multicolumn{8}{|c|}{ Specific IgE } \\
\hline & \multicolumn{2}{|c|}{$\begin{array}{l}\text { bee: positive } \\
\text { wasp: positive }\end{array}$} & \multicolumn{2}{|c|}{$\begin{array}{l}\text { bee: positive } \\
\text { wasp: negative }\end{array}$} & \multicolumn{2}{|c|}{$\begin{array}{l}\text { bee: negative } \\
\text { wasp: positive }\end{array}$} & \multicolumn{2}{|c|}{$\begin{array}{l}\text { bee: negative } \\
\text { wasp: negative }\end{array}$} \\
\hline & yes & no & yes & no & yes & no & yes & no \\
\hline \multicolumn{9}{|c|}{ Specific IgE to CCDs $>0.35 \mathrm{kU}_{\mathrm{A}} / \mathrm{l}$} \\
\hline Positive $(\mathrm{n}=15)$ & $12(80 \%)$ & $3(20 \%)$ & $1(7 \%)$ & $14(93 \%)$ & $0(0 \%)$ & $15(100 \%)$ & $2(13 \%)$ & $13(87 \%)$ \\
\hline Negative $(\mathrm{n}=2,205)$ & $28(1 \%)$ & $2,177(99 \%)$ & $32(1 \%)$ & $2,173(99 \%)$ & $263(12 \%)$ & $1,942(88 \%)$ & $1,882(85 \%)$ & $323(15 \%)$ \\
\hline Total & 40 & 2,180 & 33 & 2,187 & 263 & 1,957 & 1,884 & 336 \\
\hline Fisher's test & \multicolumn{2}{|c|}{$\mathrm{p}<0.0001$} & \multicolumn{2}{|l|}{$\mathrm{p}=0.2$} & \multicolumn{2}{|l|}{$\mathrm{p}=0.2416$} & \multicolumn{2}{|l|}{$\mathrm{p}<0.0001$} \\
\hline OR (95\% CI) & \multicolumn{2}{|c|}{311 (83->999) } & \multicolumn{2}{|c|}{$4.9(0.6-38)$} & \multicolumn{2}{|l|}{-} & \multicolumn{2}{|c|}{$0.03(0.006-0.12)$} \\
\hline
\end{tabular}

tized but with IgE to venom below the detection limit was not seen for the major bee allergen IgE assay.

Median serum tryptase was $4.8 \mu \mathrm{g} / \mathrm{l}(95 \%$ CI 2.1-11.7 $\mu \mathrm{g} / \mathrm{l})$. In total, $5.4 \%$ of the population had serum tryptase above the reference limit of $11.4 \mu \mathrm{g} / \mathrm{l}$. Elevated levels did not occur more frequently in individuals with sting reactions $(11 / 246 ; 4.5 \%)$, not even malaise $(4 / 82 ; 4.9 \%)$ compared to nonreactors $(102 / 1,844 ; 5.5 \%$; n.s $)$. For each type of reaction and for no reactions, serum tryptase concentrations did not differ when comparing individuals with or without specific IgE to venom.

\section{Discussion}

A prevalence of insect sting allergies of up to $43 \%$ has been reported in selected populations and probably varies with exposure, as determined by climate and activities [3, 9]. Few general population studies have been performed. An Austrian study [2] found large local reactions in 4.6\% and systemic reactions in $3.3 \%$, i.e. only about half the prevalence in our larger study. The methodology using telephone interviews versus administered questionnaires with only a few questions might explain some of the differences. Systemic reactions in our study were either 'malaise' and/or 'itching rash covering large parts of the body' and, most likely, 'malaise' would include some vasovagal and severe pain reactions or hyperventilation in addition to anaphylactic reactions. A large study on an adolescent subtropical population reported figures of the same magnitude as our study [10]. The explanation might be that a lower exposure level in our colder climate compensated for the longer exposure time in our adult population with an older median age. A smaller rural area study on adults [11] and a larger study among industrial
Table 4. Specific IgE to venom in individuals with or without possible allergic reactions to stinging insects

\begin{tabular}{|c|c|c|c|}
\hline & \multicolumn{2}{|c|}{ Specific IgE to wasp and/or bee } & \multirow[t]{2}{*}{ Total } \\
\hline & positive $^{1}$ & negative & \\
\hline \multicolumn{4}{|c|}{$\begin{array}{l}\text { Symptoms occurring with stings by a wasp, bee or an unknown } \\
\text { insect }^{2}\end{array}$} \\
\hline Yes & $99(5 \%)$ & $159(8 \%)$ & $258(12 \%)$ \\
\hline No & $223(11 \%)$ & $1,610(77 \%)$ & $1,833(88 \%)$ \\
\hline Total & $322(15 \%)$ & $1,769(85 \%)$ & $2,091(100 \%)$ \\
\hline
\end{tabular}

workers [12] in another subtropical area only found systemic reactions in $2.3 \%$. However, in the rural study, the proportion sensitized in the symptomatic group was only slightly higher than in our study.

We measured only IgE levels to wasp and honeybee venoms in this study. In northern Europe, the majority of stings encountered by humans would be from wasps (Vespula sp.) and honeybees (Apis mellifera). Other bees, European hornets (Vespa crabo) and paper wasps (Polistes sp.) are responsible for only a relatively small number of stings in humans in this area, and it is unlikely that stings with venom not tested for would be a major explanation for reactions in individuals with no IgE to venom. The reporting of lifetime prevalence in an adult population implies recall bias, both with respect to the type of stinging insect and to the symptoms, e.g. a painful reaction a long time ago could be remembered as larger than it actually was. Another major explanation 
for the discrepancy might be the decline in specific $\operatorname{IgE}$ over time $[13,14]$.

Less than half of the individuals with measurable IgE to venoms had a history of previous reactions. This is in the same range as found in other studies [11]. For inhalant allergies, subclinical sensitization is well known and at least $20 \%$ of individuals with IgE to seasonal allergens, such as pollen, do not have symptoms during (a normal) pollen season [15]. For venom allergies, part of the explanation could be that we are comparing previous reaction patterns with present IgE levels and not IgE levels to subsequent sting reactions. However, in a new sting challenge study performed on individuals with IgE to venom but no previous symptoms, only $5 \%$ reacted with systemic reactions, which is comparable to the general population [16]. In this study, a surprisingly high proportion (44\%) reacted with large local reactions. However, the risk for systemic reactions to future stings in large local reactors with significant amounts of specific IgE is only slightly above the risk in nonreactors, indicating that the type of previous reaction is a more important prognostic factor than is specific IgE [17]. This is why venom-specific IgE testing should not be a candidate for inclusion in general health screenings or even in investigations of most cases of large local sting reactions [17]. We had hoped that specific IgE plus serum tryptase would increase the correlation between reactors and blood tests. However, this was not the case. Prospective studies are needed to establish the true predictive value of specific $\mathrm{IgE}$ as well as serum tryptase.

In patients, it is of major importance to determine whether immunological cross reactivity to different venoms implies clinical reactions to these venoms. The frequency of clinical cross-reactions in people with symptoms is higher than the frequency of individuals with $\operatorname{IgE}$ to both bee and wasp venoms. This most likely reflects difficulties in identification of the insects [18] as well as the fact that some of the reactions were not allergic ones. IgE to the major venom allergens $\mathrm{rApi} \mathrm{m} 1$, rVes $\mathrm{v} 1$ and rVes $\mathrm{v} 5$ have been shown to have a very high sensitivity and specificity when investigating DS patients [19-22]. Our study supports the value of adding IgE to major allergens in such patients, since double sensitization could only be confirmed in $10 \%$. Sensitivity in our study was, however, much lower than in previous studies [21] with carefully selected and probably more severely affected patients. $31 \%$ of the DS individuals had no IgE to the major allergens in our population study. Increase in sensitivity of the test for IgE to Api m 1 and/or additional tests for IgE to other insect venom allergens might be useful.
IgE to major wasp allergens could document sensitization in (a few) individuals with systemic symptoms, but with IgE to wasp venom below the normal detection value. This was not the case for IgE to the major bee allergen, supporting the finding that IgE to rApi $\mathrm{m} 1$ has a lower level of sensitivity [23-25].

It has been shown that insect allergic patients with $\operatorname{IgE}$ to CCDs have an increased risk of being misdiagnosed as having pollen sensitization [26], but in general, atopy is not regarded as a major risk factor for insect allergy [1, 12]. However, our population study with an OR of 2.65.4 for sensitization to wasp and bee stings could potentially be used to modify the concept. It is unlikely that sensitivity to CCDs is responsible for this finding since the prevalence was rather low in our population. An OR of $>8$ for sensitization to wasp venom if sensitized to bee venom is, however, in favor of common allergenic component(s), i.e. cross-reactivity rather than coincidental sensitization to different allergens. With an OR of $>300$ for sensitivity to both bee and wasp venom when having IgE to CCDs, CCD structure seems to be a very important candidate for such a common component. The additional observation that several of the DS individuals with IgE to CCDs in our unselected population had, in fact, had symptoms when stung, indicates some clinical relevance of this sensitization, although in many cases it occurs with cosensitization to other venom and pollen (especially grass) allergens [27, 28].

That there was no increased reaction rate to stings in individuals with serum tryptase above the normal range may be surprising, since elevated serum tryptase has been identified as a risk factor for more severe sting reactions in case control studies [5]. However, serum tryptase seems not to be associated with atopy and allergic respiratory disease [29], and patients with indolent mastocytosis without skin lesions have been found to have a high reaction rate to insect stings but a lower baseline serum tryptase than other groups of mastocytosis patients [30].

In conclusion of this first large general population study on insect allergies in northern Europe, The majority of those sensitized did not report symptoms and the majority who reported having had symptoms were not sensitized. IgE to CCDs occurred less frequently when compared to other regions, but the occurrence was highly linked to double sensitization with and without sting reactions. Our study supported that measurement of IgE to major allergens/components in the venom has an important place in the investigation of DS patients and IgE(and skin test) negative patients with a convincing clinical history. However, 'specificity' and problems with differ- 
entiation have been reported [31] and, in a high proportion of the population, IgE to venom was neither accompanied by IgE to CCDs nor to the major allergens tested for, so it is likely that other components (still not identified) of the many allergens in the venom [32] are responsible for the sensitization. There is a need for commercially available tests for IgE to additional venom allergens to better diagnose patients with double sensitization and also to ensure that relevant allergens are present in extracts used for immunotherapy, which has not always been the case [33].

\section{Acknowledgement}

The Tryg Foundation (7-11-0213) supported The Health2006 5 -year follow-up study. We thank Thermo Fisher Scientific, ImmunoDiagnostics (formerly Phadia), Allerød, Denmark, for supporting the Health2006 5-year follow-up study with the entire laboratory testing services and ImmunoCAP reagents.

\section{Disclosure Statemernt}

There were no conflicts of interest.

\section{References}

1 Bilò BM, Bonifazi F: Epidemiology of insectvenom anaphylaxis. Curr Opin Allergy Clin Immunol 2008;8:330-337.

2 Bokanovic D, Aberer W, Griesbacher A, Sturm GJ: Prevalence of Hymenoptera venom allergy and poor adherence to immunotherapy in Austria. Allergy 2011;66:1395-1396.

3 Biló BM, Rueff F, Mosbech H, Bonifazi F, Oude-Elberink JNG, Birnbaum J, et al: Diagnosis of Hymenoptera venom allergy. Allergy 2005;60:1339-1349.

4 Kucharewicz I, Bodzenta-Lukaszyk A, Szymanski W, Mroczko B, Szmitkowski M: Basal serum tryptase level correlates with severity of hymenoptera sting and age. J Investig Allergol Clin Immunol 2007;17:65-69.

5 Ruëff F, Przybilla B, Biló MB, Müller U, Scheipl F, Aberer W, et al: Predictors of severe systemic anaphylactic reactions in patients with Hymenoptera venom allergy: importance of baseline serum tryptase - a study of the European Academy of Allergology and Clinical Immunology Interest Group on insect venom hypersensitivity. J Allergy Clin Immunol 2009;124:1047-1054.

6 Thuesen BH, Cerqueira C, Aadahl M, Ebstrup JF, Toft U, Thyssen JP, et al: Cohort profile: the Health2006 cohort, Research Centre for Prevention and Health. Int J Epidemiol 2014; 43:568-575.

7 Gonzalez-Quintela A, Dam Laursen AS, Vidal C, Skaaby T, Gude F, Linneberg A: IgE antibodies to alpha-gal in the general adult population: relationship with tick bites, atopy, and cat ownership. Clin Exp Allergy 2014;44: 1061-1068.

8 Bonadonna P, Perbellini O, Passalacqua G, Caruso B, Colarossi S, Dal Fior D, et al: Clonal mast cell disorders in patients with systemic reactions to Hymenoptera stings and increased serum tryptase levels. J Allergy Clin Immunol 2009;123:680-686.
9 Von Moos S, Graf N, Johansen P, Müllner G, Kündig TM, Senti G: Risk assessment of Hymenoptera re-sting frequency: implications for decision-making in venom immunotherapy. Int Arch Allergy Immunol 2013;160:8692.

10 Graif Y, Romano-Zelekha O, Livne I, Green MS, Shohat T: Allergic reactions to insect stings: results from a national survey of 10,000 junior high school children in Israel. J Allergy Clin Immunol 2006;117:1435-1439.

11 Fernandez J, Blanca M, Soriano V, Sanchez J, Juarez C: Epidemiological study of the prevalence of allergic reactions to Hymenoptera in a rural population in the Mediterranean area. Clin Exp Allergy 1999;29:1069-1074.

12 Navarro LA, Peláez A, de la Torre F, Burillo JMT, Megías J, Martínez I: Epidemiological factors on Hymenoptera venom allergy in a Spanish adult population. J Investig Allergol Clin Immunol 2004;14:134-141.

13 Mosbech H: Insect allergy. A comparative study including case histories and immunological parameters. Allergy 1984;39:543-549.

14 Golden DBK, Marsh DG, Freidhoff LR, Kwitterovich KA, Addison B, Kagey-Sobotka A, et al: Natural history of Hymenoptera venom sensitivity in adults. J Allergy Clin Immunol 1997;100:760-766.

15 Linneberg A, Nielsen NH, Madsen F, Frølund L, Dirksen A, Jørgensen T: Increasing prevalence of specific IgE to aeroallergens in an adult population: two cross-sectional surveys 8 years apart: the Copenhagen Allergy Study. J Allergy Clin Immunol 2000;106:247-252.

16 Sturm GJ, Kranzelbinder B, Schuster C, Sturm EM, Bokanovic D, Vollmann J, et al: Sensitization to Hymenoptera venoms is common, but systemic sting reactions are rare. J Allergy Clin Immunol 2014;133:16351643.

17 Golden DBK: Large local reactions to insect stings. J Allergy Clin Immunol Pract 2015;3: 331-334.
18 Baker TW, Forester JP, Johnson ML, Stolfi A, Stahl MC: The HIT study: Hymenoptera identification test - how accurate are people at identifying stinging insects? Ann Allergy Asthma Immunol 2014;113:267-270.

19 Mittermann I, Zidarn M, Silar M, MarkovicHousley Z, Aberer W, Korosec P, et al: Recombinant allergen-based IgE testing to distinguish bee and wasp allergy. J Allergy Clin Immunol 2010;125:1300-1307.

20 Müller UR, Johansen N, Petersen AB, Fromberg-Nielsen J, Haeberli G: Hymenoptera venom allergy: analysis of double positivity to honey bee and Vespula venom by estimation of IgE antibodies to species-specific major allergens Api $\mathrm{m} 1$ and Ves v 5. Allergy 2009;64: 543-548.

21 Müller U, Schmid-Grendelmeier P, Hausmann $\mathrm{O}$, Helbling A: IgE to recombinant allergens Api m 1, Ves v 1, and Ves v 5 distinguish double sensitization from cross-reaction in venom allergy. Allergy 2012;67: 1069-1073.

22 Seismann H, Blank S, Cifuentes L, Braren I, Bredehorst R, Grunwald T, et al: Recombinant phospholipase A1 (Ves v 1) from yellow jacket venom for improved diagnosis of Hymenoptera venom hypersensitivity. Clin $\mathrm{Mol}$ Allergy 2010;8:7.

23 Korošec P, Valenta R, Mittermann I, Čelesnik $\mathrm{N}$, Eržen R, Zidarn M, et al: Low sensitivity of commercially available rApi $\mathrm{m} 1$ for diagnosis of honeybee venom allergy. J Allergy Clin Immunol 2011;128:671-673.

24 Sturm GJ, Hemmer W, Hawranek T, Lang R, Ollert M, Spillner E, et al: Detection of IgE to recombinant Api $\mathrm{m} 1$ and $\mathrm{rVes} \mathrm{v} 5$ is valuable but not sufficient to distinguish bee from wasp venom allergy. J Allergy Clin Immunol 2011;128:247-248. 
25 Sturm GJ, Biló MB, Bonadonna P, Hemmer W, Caruso B, Bokanovic D, et al: Ves v 5 can establish the diagnosis in patients without detectable specific IgE to wasp venom and a possible north-south difference in Api $m 1$ sensitization in Europe. J Allergy Clin Immunol 2012;130:817.

26 Kochuyt A-M, Van Hoeyveld EM, Stevens EAM: Prevalence and clinical relevance of specific immunoglobulin $\mathrm{E}$ to pollen caused by sting-induced specific immunoglobulin $\mathrm{E}$ to cross-reacting carbohydrate determinants in Hymenoptera venoms. Clin Exp Allergy 2005;35:441-447.

27 Hofmann SC, Pfender N, Weckesser S, HussMarp J, Jakob T: Added value of IgE detection to rApi $\mathrm{m} 1$ and rVes $\mathrm{v} 5$ in patients with $\mathrm{Hy}$ menoptera venom allergy. J Allergy Clin Immunol 2011;127:265-267.
28 Eberlein B, Krischan L, Darsow U, Ollert M, Ring J: Double positivity to bee and wasp venom: improved diagnostic procedure by recombinant allergen-based IgE testing and basophil activation test including data about cross-reactive carbohydrate determinants. J Allergy Clin Immunol 2012;130:155-161.

29 Fenger RV, Linneberg A, Vidal C, Vizcaino L, Husemoen LL, Aadahl M, et al: Determinants of serum tryptase in a general population: the relationship of serum tryptase to obesity and asthma. Int Arch Allergy Immunol 2012;157: 151-158.
30 Alvarez-Twose I, Zanotti R, González-deOlano D, Bonadonna P, Vega A, Matito A, et al.: Nonaggressive systemic mastocytosis (SM) without skin lesions associated with insect-induced anaphylaxis shows unique features versus other indolent SM. J Allergy Clin Immunol 2014;133:520-528.

31 Sturm GJ, Jin C, Kranzelbinder B, Hemmer W, Sturm EM, Griesbacher A, et al: Inconsistent results of diagnostic tools hamper the differentiation between bee and vespid venom allergy. PLoS One 2011;6:1-8.

32 Hoffman DR: Structural biology of allergens from stinging and biting insects. Curr Opin Allergy Clin Immunol 2008;8:338-342.

33 Köhler J, Blank S, Müller S, Bantleon F, Frick M, Huss-Marp J, et al: Component resolution reveals additional major allergens in patients with honeybee venom allergy. J Allergy Clin Immunol 2014;133:1383-1389. 